\title{
MULTI-DETECTOR CT CHARACTERIZATION OF CORONARY ATHEROMATOUS PLAQUES
}

\author{
Marwa Maher; Ahmed Samy; Islam Shawky; Waleed Hetta and Wahid Tantawy
}

Radiology department Ain shams
University, ${ }^{*}$ Radiology department,
Police Hospital, Cairo, Egypt
Corresponding author
Marwa Maher Ahmed Hafez
Mobile: +201000373300
E.mail:
maher.marwa@ gmail.com.

Received: $25 / 2 / 2021$

Accepted: $26 / 3 / 2021$

Online ISSN: $2735-3540$

\begin{abstract}
:
Background: Atherosclerotic cardiovascular diseases are the leading cause of death worldwide (30\% of the mortalities).

Coronary atherosclerotic plaques are classified into stable and unstable. Unstable plaques increase the risk of acute coronary syndrome and early detection will help prevention. Evaluation of plaque vulnerability is done best by invasive intravascular ultrasound and so, this study aims to evaluate the possible use of non invacive CT for plaque characterization.
\end{abstract}

Aim of the work: Determine the role of multidetector CT in the assessment of plaque composition and configuration. This will improve risk stratification thus, decreasing the incidence of sudden death.

Patients and methods: This study included 46 patients with coronary atherosclerotic plaques. The most expansive or solitary lesion was selected for each patient.

Results: Forty-six atherosclerotic plaques were evaluated by multi-slice coronary CT; 30 patients were unstable clinically and 16were stable. Dyslipidemia was found in 40 patients while diabetes was found in 22 patients. $69.6 \%$ of the lesions were found in the proximal LAD. Most of unstable patients showed mixed plaques and soft plaques. In a stable group, most of the plaques were calcified. As for the attenuation pattern 18/46 of the plaque with heterogeneous attenuation were in the unstable clinical group. 28/46 of the plaques with homogenous attenuation were stable. Regarding the Napkin ring sign it was positive in 18/46 of the plaques and the 18 patients on follow up were found to be unstable.

All patients with spotty calcification proved to be unstable clinically.

Remodeling index, low hounse field unit and plaque burden were higher in the unstable group.. No association between stability and degree of stenosis.

Conclusion: A strong correlation between plaque characteristics and clinical presentation: Multislice coronary CT angiography detect high-risk plaque criteria.

Keywords: Remodeling index, Napkin ring, High-risk plaque, Hounsfield unit, spotty calcification plaque attenuation pattern and plaque burden.

\section{INTRODUCTION:}

Coronary plaque disruption and subsequent thrombosis make coronary atherosclerosis a potentially life-threatening disease. The risk of plaque rupture seems to depend on plaque composition rather than on plaque volume. Most ruptures occur in plaques containing a soft, lipid-rich core that is covered by a thin fibrous cap. Small ruptures often remain clinically silent, but more extensive plaque ruptures may cause 
the onset of unstable angina, myocardial infarction or sudden death ${ }^{1}$.

Intravascular ultrasound (IVUS) is the most established invasive method with a long clinical track record. Besides, the spectral analysis of IVUS-derived radiofrequency $(\mathrm{RF})$ data enables a more precise analysis of the plaque composition and plaque-type.

MDCT is seen as a potential alternative to current imaging methods for assessing vessel anatomy and atherosclerotic plaque composition/morphology in a great variety of arterial beds ${ }^{2}$.

\section{PATIENTS AND METHODS:}

This study was approved by the ethics committee of our institution. This study carried out on 46 patients done at police hospital Nasr city in December 2017 and June 2020.

Study population: Patients subjected to the study according to the following criteria:

\section{Inclusion criteria:}

Both genders and all ages were included. Patients with coronary atherosclerotic plaques with normal LV systolic function for all studied patients. The most expansive or solitary plaque lesion was selected for each patient.

\section{Exclusion criteria:}

Emergency patients with unstable angina that required immediate intervention, High coronary calcium score (more than 400),Pregnancy, Patient with previous percutaneous coronary intervention (PCI) or coronary artery bypass graft (CABG), Sensitivity to contrast media and Patients with chronic renal failure not on regular dialysis.

\section{Patient preparation:}

NPO 4hours, No caffeine for 24 hours, Beta-blocker an hour before the study No
Viagra or for 7 days before the study, Oral sedation diazepam $5 \mathrm{mg}$ in anxious patients, ECG check and lead fixation, Sublingual nitroglycerin spray or tab. A small exercise to train the patient to sustain a breath-hold long enough and again checking the potential heart rate variability during the beath hold period. In cases with wide heart rate variations or with frequent premature contractions during apnea, administration of oxygen through nasal cannulae during acquisition to reduce these events maintain a stable heart rate. A stable venous line must be available requiring an 18-20 gauge needle placed in the antecubital vein

\section{CT Imaging Technique:}

Patients were scanned using 320-row MDCT scanner (Aquillion One, Toshiba Medical Systems, Otawara, Japan). The investigation is started primarily with scanogram to adjust the scanning range of coronary CTA, then non-contrast calcium scoring. The acquired images were transferred to the off-line workstations (Toshiba Vitrea Medical System ).

The amount of contrast required is $65 \mathrm{cc}$ of low osmolar contrast media. The rate of contrast administration should be rapid at a rate of 4 to $5 \mathrm{cc} / \mathrm{s}$ for proper enhancement.

\section{Acquisition Parameters:}

$0.35 \mathrm{~s}$ gantry rotation time, $\mathrm{mA}$ is automatically by the scanner according to patient body habitus (range 300-580 mA), variable $\mathrm{kV}$ according to body habitus. A breathing exercise trial takes place to assess the heart rate during the breath-holding period of $10 \mathrm{~s}$ after which the scanner automatically adjusted the exposure window settings for optimal temporal resolution. Prospective ECG-triggered acquisition is used for patients with a heart rate below 60 bpm exposing only 70-80\% of R-R interval. For those with higher heart rate, the acquisition was done by widening the exposure window to include $30-80 \%$ of R-R interval. During the rest of the R-R interval, 
X-ray is switched off completely by the scanner to decrease radiation exposure.

\section{Image Reconstruction:}

Images reconstruction is done with a slice thickness of $0.5 \mathrm{~mm}$ with $0.3 \mathrm{~mm}$ interval. The best phase in diastole and using soft and sharp reconstruction kernel at $75 \%$ of R-R interval. The best systolic phase was reconstructed also if the exposure window included $30-80 \%$ of R-R interval.

\section{Post Processing:}

The reconstructed images were transferred to a workstation (Vitrea FX, Vital Images, USA) to review axial images and also to obtain multiplanar reformatted images at sagittal and coronal planes. Also, maximum intensity projection images, 3D volume-rendered images, semitransparent 3D volume-rendered images, and curved planar reformations were obtained for a detailed assessment of coronary plaques.

\section{Image analysis:}

All coronary computed tomography angiography (CCTA) scans were evaluated for the presence of nonvaluable segments. Coronary arteries were divided into 17 segments according to the American Heart Association Classification. Coronary plaques were defined as structures at least $1 \mathrm{~mm} 2$ within and/or adjacent to artery lumen, clearly distinguishable from the vessel lumen and surrounded by pericardial tissue. Quantitative CTA lesion analysis was performed on all plaques $>25 \%$ by automated software that automatically segments vessel margins, separate different plaque components by using attenuation thresholds (30-60 for lipid plaque, 61-149 for fibrous plaque and 150-1300 for calcium) and provide a colour map of plaque compositions. CT density was screened with pixels and Hounsfield unit (HU) was recorded; the area of pixels with predetermined attenuation threshold was calculated via semiautomated quantitative analysis and plaque attenuation is represented with mean and standard deviation. Degree of stenosis was defined as referential diameter-luminal diameter/ referential diameter. In turn, the referential diameter was defined as the diameter located in the proximal normal vessel or distal normal vessel if the lesion is ostial. The stenosis severity is graded as minimal < $10 \%$ diamter or area stenosis, mild $\leq 49 \%$, moderate $50-69 \%$, severe $\geq 70 \%$, subtotal $>$ $90 \%$, and total occlusion per coronary segment. All plaques > 25\% stenoses were analyzed for morphologic features. Parameters analyzed including plaque burden of the whole plaque and at an area of maximal narrowing = (lesion plaque area lesion lumen area/lesion plaque area), low attenuation plaque (LAP); CT density was recorded with quantitative CTA analysis and the area of a region of interest (ROI) LAP is defined as hypoattenuation with CT density < $30 \mathrm{HU}$ ). NRS was defined as an outer high-density rim with the inner hypodense area not adjacent to calcification and present on a minimum of two adjacent axial $1 \mathrm{~mm}$ slices, and spotty calcification was defined as calcification $<3 \mathrm{~mm}$. The plaques are divided into stable group and unstable groups radiologically. The unstable group showed one or more of the following factors: low mean Hounsfield units (30HU), spotty calcification $(<3 \mathrm{~mm})$, positive remodelling index $>1.05$, increase plaque burden above 69\%, heterogeneous attenuation, soft or mixed (30-60) and (61149)composition and positive Napkin ring sign. The stable group did not show any of these factors.

\section{Clinical Follow up for comparison:}

After a year of clinical Follow up to both groups patients were classified clinically into stable and unstable groups and their clinical data was compared to their radiological data.

Clinically Stable patients showed atypical chest pain and stable angina (class I angina; anginal pain with prolonged effort 
relieved by rest and class II angina; mild inadequacy of normal daily activity)The clinically unstable group consist of patients with class III angina; severe inadequacy of ordinary daily activity and class IV; chest discomfort on any daily activity, and patients with major adverse cardiac events (non-ST segment elevated myocardial infarction NSTEMI or STEMI) that relieved medically (diagnosed by the referring doctor, follow up discharge card, or patients admitted to coronary care unit).

\section{Statistical analysis:}

Statistical analysis was performed using MedCalc statistical software for Windows (MedCalc Software, Mariakerke, Belgium). Data for continuous variables were expressed as either median, interquartile range and range or mean \pm standard deviation and as both number and percentage for categorical data. Mann Whitney and the independent-samples T-test were used to evaluate the differences in quantitative variables when the data was not normally and normally distributed respectively. Categorical data were evaluated using the Chi-squared test. Receiver operator characteristic (ROC) curve analysis was performed to determine the diagnostic accuracy of the various variables in distinguishing the different groups. The diagnostic accuracy of all variables was evaluated in terms of sensitivity, specificity, positive predictive value (PPV), negative predictive value (NPV), and area under the ROC curve (AUC). For all tests all $\mathrm{P}$ values were twotailed and a $\mathrm{P}$-value $<0.05$ was considered significant.

\section{RESULTS:}

The study included 46 patients: 16 were with stable coronary artery disease and 30 were with unstable coronary artery disease, 34 were males while 12 patients were females. The mean age population was 56.6 \pm 7.71 years. In comparing results obtained radiologically with clinical follow-up Remodeling index was higher in the unstable group with a median of 1.4. As well as the plaque burden which was also higher in the unstable group. Plaques in the unstable clinical group also showed low hounse field units while the stable group showed a lot higher hounse field units. There was not any association between neither Stability nor the degree of stenosis. The study showed that Napkin ring sign and plaque attenuation pattern both had the largest AUC followed by plaque-type and finally spotty calcifications. However, Napkin ring sign and plaque attenuation pattern were better than spotty calcifications in the differentiation of unstable and stable plaques. No significant difference was seen between the AUCs of plaque-type and spotty calcifications.

Performance of the various parameters (plaque attenuation pattern, spotty calcification napkin ring sign and the type of the plaque; soft/mixed versus calcific) in the differentiation of unstable plaques from stable plaques, the number of true positive (TP), true negative (TN), false positive (FP), and false-negative (FN) cases, as well as the calculated sensitivities, specificities, positive predictive values (PPV), negative predictive values (NPV), accuracies, the area under curves (AUC) and their associated $\mathrm{P}$ values of all parameters, are listed in Table 1. 
Table (1)

\begin{tabular}{|l|c|c|c|c|c|c|c|c|c|c|c|}
\hline Parameter & TP & TN & FP & FN & Sensitivity & Specificity & PPV & NPV & Accuracy & AUC & P-value \\
\hline $\begin{array}{l}\text { Plaque att. } \\
\text { Pattern }\end{array}$ & 18 & 16 & 0 & 12 & $60 \%$ & $100 \%$ & $100 \%$ & $57.1 \%$ & $73.9 \%$ & 0.80 & $<0.0001$ \\
\hline $\begin{array}{l}\text { Plaque type } \\
\text { soft/mixed } \\
\text { vs calcific }\end{array}$ & 26 & 10 & 6 & 4 & $86.7 \%$ & $62.5 \%$ & $81.3 \%$ & $71.4 \%$ & $78.3 \%$ & 0.75 & 0.0004 \\
\hline $\begin{array}{l}\text { Spotty } \\
\text { calcificatio } \\
\text { ns }\end{array}$ & 8 & 16 & 0 & 22 & $26.7 \%$ & $100 \%$ & $100 \%$ & $42.1 \%$ & $52.2 \%$ & 0.63 & 0.001 \\
\hline $\begin{array}{l}\text { Napkin ring } \\
\text { sig n }\end{array}$ & 18 & 16 & 0 & 12 & $60 \%$ & $100 \%$ & $100 \%$ & $57.1 \%$ & $73.9 \%$ & 0.80 & $<0.0001$ \\
\hline
\end{tabular}

To arrange the variables according to their diagnostic performance in the differentiation of unstable plaques from stable plaques, the area under their ROC curves were compared. Napkin ring sign and plaque attenuation pattern both had the largest AUC followed by plaque-type and finally spotty calcifications (Figure 1). Though the AUCs of plaque attenuation pattern and napkin ring sign were greater than that of plaque-type, no significant difference was found between their respective AUCs indicating that the diagnostic performance of these parameters did not differ significantly. However, the AUCs of Napkin ring sign and plaque attenuation pattern were significantly greater than the AUC of spotty calcification indicating that Napkin ring sign and plaque attenuation pattern were better than spotty calcifications in the differentiation of unstable and stable plaques. No significant difference was seen between the AUCs of plaque-type and spotty calcifications Details of the pairwise comparisons of all ROC curves and their statistical significance are listed in Table 2.

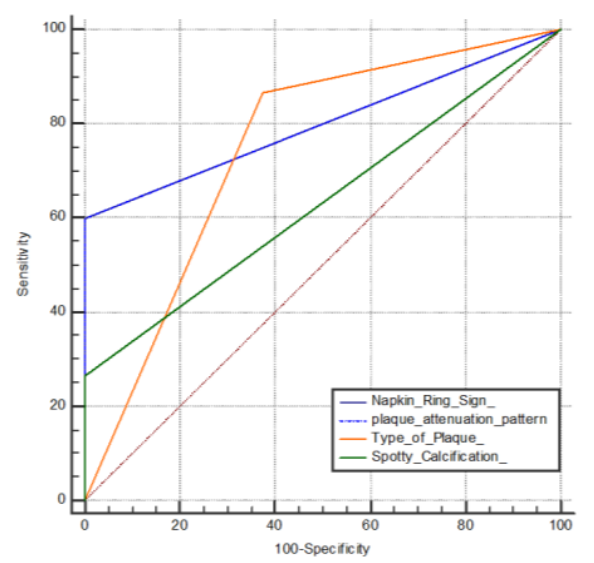

Figure 1: Receiver operating characteristic curve analyses. The specificity and sensitivity of using plaque attenuation pattern, spotty calcification, napkin ring sign and the type of the plaque; soft/mixed versus calcific in the differentiation of unstable plaques from stable plaques were compared. Plaque attenuation pattern and napkin ring sign had equal AUCs and no significant differences were seen between either of their AUCs and that of plaque-type or between the AUC of plaque-type and spotty calcifications, yet the AUCs of plaque attenuation and napkin ring sign were significantly greater than the AUC of spotty calcifications. 
Table 2: Difference between the ROC curves of all parameters and their statistical significance

\begin{tabular}{|l|c|c|c|c|c|c|c|c|}
\hline & \multicolumn{2}{|c|}{$\begin{array}{c}\text { Plaque att. } \\
\text { pattern }\end{array}$} & \multicolumn{2}{c|}{ Napkin ring sign } & \multicolumn{2}{c|}{ Type of plaque } & \multicolumn{2}{c|}{$\begin{array}{c}\text { Spotty } \\
\text { calcifications }\end{array}$} \\
\hline & Diff $^{\text {a }}$ & $\begin{array}{c}\text { P- } \\
\text { value }\end{array}$ & Diff $^{\text {a }}$ & P-value & Diff $^{\text {a }}$ & P-value & Diff $^{\text {a }}$ & P-value \\
\hline Plaque att. Pattern & - & - & 0.000 & $\begin{array}{c}1.000 \\
\text { (NS) }\end{array}$ & 0.054 & $\begin{array}{c}0.469 \\
\text { (NS) }\end{array}$ & 0.167 & 0.010 (S) \\
\hline Napkin ring sign & - & - & - & - & 0.054 & $\begin{array}{c}0.469 \\
\text { (NS) }\end{array}$ & 0.167 & $0.010(\mathrm{~S})$ \\
\hline Type of plaque & - & - & - & - & - & - & 0.112 & $\begin{array}{c}0.146 \\
\text { (NS) }\end{array}$ \\
\hline
\end{tabular}

adifference between the ROC curves
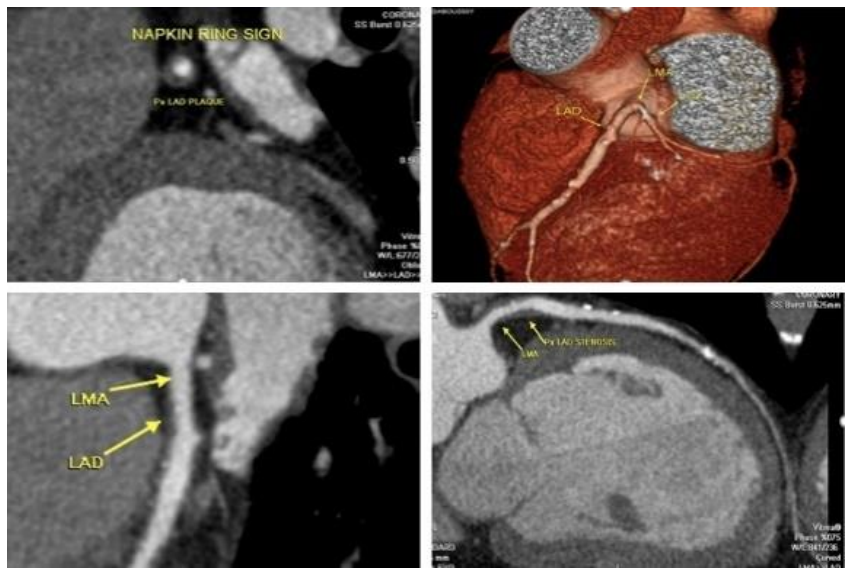

Figure 2: male patient 64-year old smoker and hypertensive. Findings: Lad the proximal lad shows an eccentric faintly calcified vulnerable plaque (as it shows napkin ring sign \& spots of calcification) causing mild stenosis. The rest of the LAD \& its branches show mild atherosclerotic changes with no significant stenosis.
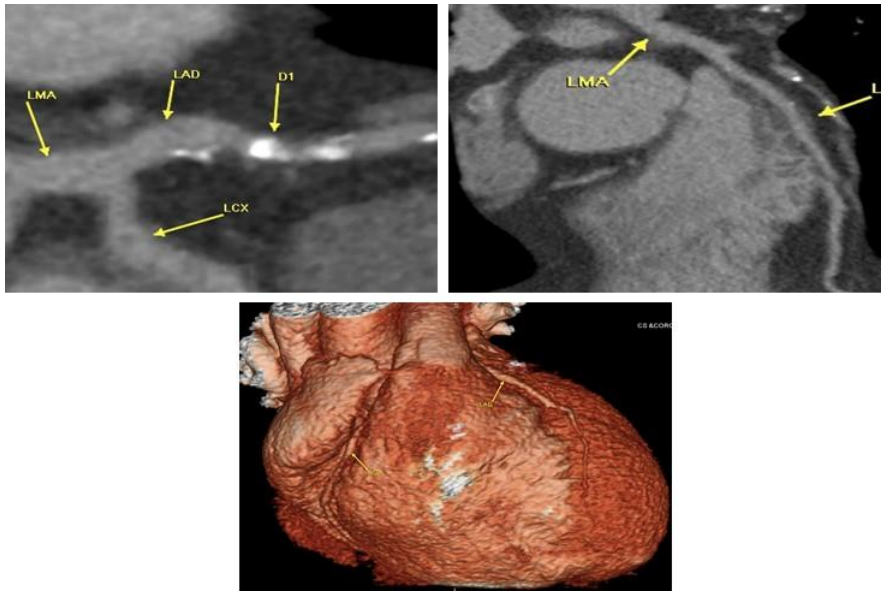

Figure3: male patient 70-years old smoker with high lipid profile. Findings: A long tortuous artery that reaches the apex. The LAD supplies two diagonal branches (D1 is an early branch) and many septal perforators. The mid LAD shows an eccentric non-calcified vulnerable plaque (as it shows positive remodelling and the average attenuation of the fibro-atheromatous core is less than $30 \mathrm{HU}$ ) causing mild stenosis, followed by a long superficial myocardial bridge with non-significant calibre attenuation (its length $=23 \mathrm{~mm}$, its depth $=1 \mathrm{~mm}$ ). The rest of the LAD and its branches (more noted at the D1) show mild atherosclerotic changes with no significant stenotic lesions. 


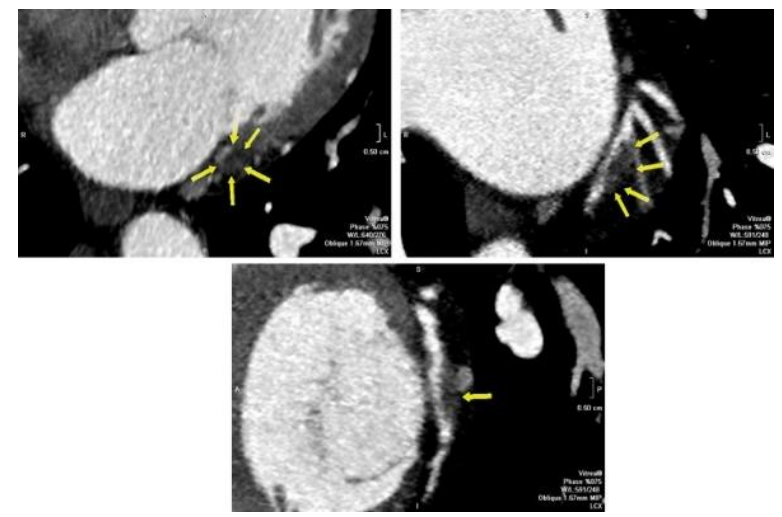

Figure 4: Male smoker patient 69-year-old with Hypertension findings: Co-dominant artery that supplies an early small high lateral, three OM branches and a PLA \& small PDA. The distal LCX after the origin of the OM3 shows a long $15 \mathrm{~mm}$ eccentric non-calcified vulnerable plaque (as it shows marked positive remodeling \& the mean attenuation of the atheromatous core is less than $30 \mathrm{HU}$ ) causing moderate to severe stenosis, followed by significant stenosis. and its branches show mild atherosclerotic changes with no significant stenosis.

\section{DISCUSSION:}

Computed Tomography Coronary Angiography (CTCA) is a promising noninvasive imaging modality, which permits the identification of HRP, including spotty calcifications, low HU attenuation plaques and napkin-ring $\operatorname{sign}^{3}$.

In this study, the number of males was significantly higher than the number of females denoting that males are more prone to CAD. This was also revealed in a study done by ${ }^{4}$ revealed that men traditionally have a higher prevalence of CAD.

Smokers were more prevalent in the unstable group in comparison to nonsmokers $(\mathrm{P}=0.004)$. This was similar research in the 1950 by $^{5}$ reported a strong association between cigarette smoke exposure and heart disease. Persons who consume more than 20 cigarettes daily have a 2- to 3-fold increase in total heart disease.

There was a significant association between hypertension and plaque stability where hypertensive patients were prevalent in the unstable group as opposed to normotensive patients which were equally prevalent in both groups $(\mathrm{P}=0.008)$. A study by ${ }^{6}$ mentioned that high blood pressure is a major modifiable risk factor for all clinical manifestations of CAD.

There was also a significant association between hyperlipidemia and plaque stability where hyperlipidemic patients were prevalent in the unstable group as opposed to non - hyperlipidemic patients which were more prevalent in the stable group $(\mathrm{P}=$ 0.004). This highlights the underlying role of these chronic medical conditions in CAD. These results were similar to research done by ${ }^{7}$ which mentioned that diagnosing and managing hyperlipidemia as a way to prevent cardiovascular disease (CVD) is a common activity for primary care physicians.

In this study patients ' ages ranged from 41 to 74 years old. This age group was similar to the National Heart, Lung, and Blood Institute Trusted Source, which mentioned that the risk for women increases at age 55. The risk for men increases at age 45. And most of the cases in this research were between the same age group.

Besides, there was no significant association found between diabetes, obesity 
Positive family history and plaque stability $(\mathrm{P}=0.31)$ as well as the non-significant association found between family history of CAD and plaque stability $(\mathrm{p}=0.87)$. This was different from ${ }^{5}$ research of risk factors in which she mentioned that diabetes and obesity are risk factors for CAD

The HRP criteria proposed for investigation in this study were the napkinring sign (NRS), the positive remodelling, plaque composition and the spotty calcifications (SC) with heterogeneous attenuation pattern measured by plaque 'area ROI' (region of interest) or by 'pixel lens' screening techniques ${ }^{8}$.

NRS is a result of the difference in CT value between a lipid-rich necrotic core (i.e., the central low-density area) and the fibrous plaque tissue or thin fibrous cap (i.e., the peripheral high-density rim) within a plaque, which explains why the plaques lacking NRS appeared as uniform low-density ${ }^{9}$. In this study, $57 \%$ of the unstable plaques expressed positive NRS yet, the NRS was not detected in the remaining $43 \%$ of unstable plaques. On the other hand, $100 \%$ of the stable plaques revealed negative NRS. Similar results were observed in an ex-vivo study done by ${ }^{10}$ revealed that NRS plaques have outstanding specificity and low sensitivity $(98.9 \%, 24.4 \%$, respectively) in predicting plaques with a large necrotic core, a key feature of rupture-prone TCFA's.

The plaque CT values in this study showed that the unstable group had much lower CT values than the stable group. The median plaque attenuation of the unstable plaques (57.04 HU) was much lower than that of the stable plaques which in most cases was calcified (578 HU). Some overlap might exist between stable and unstable groups. These values are comparable to the values measured by ${ }^{11}$ whose results of a previous ex vivo coronary artery study revealed that the mean $\mathrm{CT}$ density of "lipidrich" or "soft" plaques was 14-49 HU and the mean attenuations within "fibrous" or "dense" lesions were between 70 and 104 HU. The variance of plaque CT values might be explained by the following facts: the CT value of plaques was highly dependent on slice width; the inclusion criteria in various studies had no uniform standards, and different coronary plaque-related studies used different CT scanners. This might provide an important reference with high accuracy to differentiate unstable plaques from stable plaques in the clinical setting.

Other prospective clinical trial organized by Otsuka et al., (2016) revealed that the optimal combined threshold of the NRS and/or the plaque CT value $\leq 53 \mathrm{HU}$ may be a good indicator to predict the unstable plaques in patients with $\mathrm{CAD}^{11}$.

Coronary CTA is also used to calculate the remodelling index. This index is "calculated as the vessel cross-sectional area at the level of the maximal stenosis divided by the average of the proximal and distal reference sites" cross-sectional areas ${ }^{8}$. In this study, no significant difference was found between the remodelling index in both groups $(P=0.49)$. In the stable group the remodelling index ranged from $1.02 \%$ $1.52 \%$ with a median of $1.25 \%$ while in the unstable group, the remodelling index ranged from $0.86 \%-2.78 \%$ with a median of $1.4 \%$. Remodelling index was higher in the unstable patient group but not significant (due to the high prevalence of calcified and mixed plaques with predominate calcified portions that were usually associated with positive remodelling. Those findings agreed with what was suggested by ${ }^{12}$ who stated that remodelling index was higher in patients who had experienced major adverse cardiac events but no significant predictive due to inclusion of calcified nodules which appear larger on CTA (positive remodelling).

A clinical trial by ${ }^{13}$ aiming to determine which HRP features are associated with ACS using IVUS definitions, found that all HRP features, except the remodelling index, were significantly associated with culprit 


\section{Multi-Detector CT Characterization of Coronary Atheromatous Plaques}

plaques in a univariable analysis among 207 coronary plaques within 32 ACS patients. In his study, he mentioned that the remodelling index was the weakest predictor of ACS in his analysis.

The degree of stenosis can be graded with coronary CTA based on guidelines published by the Society of Cardiovascular Computed Tomography (SCCT). It is graded as follows: minimal stenosis $(<25 \%$ stenosis), mild stenosis $(25 \%$ to $49 \%$ stenosis), moderate stenosis (50\% to $69 \%$ stenosis), severe stenosis $(70 \%$ to $99 \%$ stenosis) and occluded stenosis ${ }^{10}$.

In this study, no significant difference was found between the degree of stenosis in both groups $(P=0.29)$. In the stable group the stenosis ranged from $0.18 \%-74 \%$ with a median of $46.5 \%$ while in the unstable group, stenosis ranged from $12 \%-87 \%$ with a median of $53 \%$. This lack of correlation was also observed in other studies ${ }^{9,11}$.

In the current study, there was a significant association between the stability of the plaque and the nature of the plaque where a significantly larger proportion of soft and mixed plaques were unstable as opposed to calcific plaques which were more commonly stable $(\mathrm{P}=0.002)$. Cheng et al., $(2011)^{14}$ found similar results when investigating the prognostic effect of plaque composition on the 5-year mortality rate based on suspected CAD patients. Morise clinical score, a simple clinical risk score, for the assessment of CAD showed that the majority of patients with calcified plaques are of low to intermediate pretest risk for CAD.

Coronary artery calcium score offers additional prognostic information but only in patients with intermediate to high CACS ${ }^{15}$. In the present study, the CACS did not influence the stability of the plaque.

Bittencourt et al., (2014) $)^{16}$ mentioned that among patients with nonobstructive $\mathrm{CAD}$, those with extensive plaque burden experienced a higher rate of cardiovascular death or myocardial infarction, comparable with those who have non-extensive plaque burden.

In this study, the plaque burden was obtained by calculating the ratio of lesion plaque area then subtracting the lesion lumen area. The stable group had a lower plaque burden $(0.26-0.79$, median 0.54$)$ than the unstable group $(0.39-0.91$ median $0.65)$, especially those patients with more than one plaque who had relatively high plaque burdens. The PROSPECT trial concluded that the strongest predictor of future events was plaque burden $\geq 70 \%$. Similar results were obtained by using CT attenuation based tissue characterization ${ }^{10}$.

The attenuation pattern is used to categorize NCP as homogeneous or heterogeneous. Heterogeneous plaques are those with two regions or more with different attenuations values, while homogeneous plaques don't show any visual differentiation. Heterogenous plaques are further classified from cross-sectional images, heterogeneous plaques with NRS and ones without.

In the current study, the different types of plaques in both groups were studied. There was a significant association between the stability of the plaque and the attenuation pattern of the plaque where heterogeneous plaques were always unstable as opposed to homogenous plaques which could be stable or unstable $(\mathrm{P}=0.0001)$.

Spotty calcification is another indicator for "vulnerable plaques. As. Several histological studies have shown that 2-7\% of sudden death cases had calcified nodules. Intra-plaque microcalcifications are thought to destabilize plaques and promote plaque rupture. In this study, there was a significant association between the presence of spotty calcifications within the plaque and the stability of the plaque where $100 \%$ of the 
patients with spotty calcification had unstable plaques $(\mathrm{P}=0.025)$.

Van Velzen et al., (2011) ${ }^{17}$ found that small spotty calcifications were noticed more in cases with TCFA's when compared to large spotty calcifications ( $31 \%$ vs. $9 \%$; $\mathrm{P}<0.05)$. These results reinforced the suggestion, that small calcified nodules are markers of high-risk plaques.

In this study, most of the plaques noted were located in the LAD (32 plaques). Consistently, these findings are in line with the study done by Taruya et al., $(2020)^{18}$ as they reported that plaques were more prevalent at the LAD especially at its proximal segment.

\section{Conclusion:}

MDCT is a great noninvasive option for characterization of coronary atheromatous plaque it can quantify; and, to a certain extent, characterize coronary atherosclerotic plaque. It can predict and so prevent ACS. Imaging findings associated with vulnerable plaque include spotty calcification, presence of napkin ring sign low mean Hounsfield units heterogenous attenuation soft tissue plaque composition as well as positive remodelling. All these factors had a lot of contribution in predicting plaque vulnerability thus facilitating a more rapid medical action.

\section{REFERENCES}

1. Schroeder S, Kopp AF, Baumbach A, et al. 2001Noninvasive detection and evaluation of atherosclerotic coronary plaques with multislice computed tomography. J Am Coll Cardiol. 37(5):1430-1435. doi:10.1016/s 0735-1097(01)01115-9

2. Cordeiro MAS, Lima JAC. 2006 Atherosclerotic Plaque Characterization by Multidetector Row Computed Tomography Angiography. J Am Coll Cardiol. 47(8 SUPPL.): C40-7. doi:10.1016/j.jacc. 2005. 09.076

3. Williams MC, Kwiecinski J, Doris M, et al.
2020 Low-Attenuation Noncalcified Plaque on Coronary Computed Tomography Angiography Predicts Myocardial Infarction: Results from the Multicenter SCOT-HEART Trial (Scottish Computed Tomography of the HEART). Circulation. 141 (18): 1452-1462. doi:10.1161/ CIRCULATIONAHA.119.044720

4. Huang Y, Hui Q, Gwinn M, et al.2020 Sexual Differences in Genetic Predisposition of Coronary Artery Disease. Circ Genomic Precis Med. CIRCGEN. 120.003147. doi:10.1161/CIRCGEN. 120. 003147

5. Malakar AK, Choudhury D, Halder B, Paul P, Uddin A, Chakraborty S. A 2019review on coronary artery disease, its risk factors, and therapeutics. J Cell Physiol. 234(10): 16812-16823. doi:10.1002/jcp.28350

6. Weber T, Lang I, Zweiker R, et al.2016 Hypertension and coronary artery disease: epidemiology, physiology, effects of treatment, and recommendations: A joint scientific statement from the Austrian Society of Cardiology and the Austrian Society of Hypertension. Wien Klin Wochenschr. 128(13-14):467-479. doi:10. 1007/ s00508-016-0998-5

7. Nelson RH. 2013Hyperlipidemia as a Risk Factor for Cardiovascular Disease. Prim Care - Clin Off Pract. 40(1):195-211. doi:10.1016/j.pop.2012.11.003

8. Oraby AS, Alarabawy RA, Abd Alla TM, Dawoud MM. 2020High risk plaque criteria by multislice coronary $\mathrm{CT}$ angiography in patients with stable vs. unstable coronary artery disease: analytic cross-sectional study. Egypt J Radiol Nucl Med. 51(1):21. doi:10.1186/s43055-020-0136-2

9. Bakhshi H, Meyghani Z, Kishi S, et al. 2019Comparative Effectiveness of CTDerived Atherosclerotic Plaque Metrics for Predicting Myocardial Ischemia. JACC Cardiovasc Imaging. 12(7):1367-1376. doi:10.1016/j.jcmg.2018.05.019

10. Kolossváry M, Szilveszter B, Merkely B, Maurovich-Horvat P.2017 Plaque imaging with CT-A comprehensive review on coronary CT angiography based risk assessment. Cardiovasc Diagn Ther. 7(5):489-506. doi:10.21037/cdt.2016.11.06 
11. Song FX, Zhou J, Zhou JJ, et al. 2018The diagnosis of coronary plaque stability by multi-slice computed tomography coronary angiography. J Thorac Dis. 10(4):23652376. doi:10.21037/jtd.2018.04.43

12. Van Rosendael AR, Narula J, Lin FY, et al.2020 Association of High-Density Calcified 1K Plaque with Risk of Acute Coronary Syndrome. JAMA Cardiol. 5(3): 282-290. doi:10.1001/jamacardio. 2019. 5315

13. Bittner DO, Mayrhofer T, Puchner SB, et al.2018 Coronary Computed Tomography Angiography-Specific Definitions of HighRisk Plaque Features Improve Detection of Acute Coronary Syndrome. Circ Cardiovasc Imaging. 11(8):e007657. doi: 10.1161/CIRCIMAGING.118.007657

14. Cheng VY, Berman DS, Rozanski A, et al.2011 Performance of the traditional age, sex, and angina typicality-based approach for estimating pretest probability of angiographically significant coronary artery disease in patients undergoing coronary computed tomographic angiography: Results from the multinational coronary CT angiography evaluation for clinical outcomes: An international multicenter registry (CONFIRM). Circulation. 124(22):
2423-2432. doi:10.1161/ CIRCULATION AHA. 111.039255

15. Stefanadis C, Antoniou CK, Tsiachris D, Pietri P. 2017Coronary atherosclerotic vulnerable plaque: Current perspectives. $J$ Am Heart Assoc. 6(3). doi:10. 1161/JAHA.117.005543

16. Bittencourt MS, Hulten E, Ghoshhajra B, et al.2014 Prognostic value of nonobstructive and obstructive coronary artery disease detected by coronary computed tomography angiography to identify cardiovascular events. Circ Cardiovasc Imaging. 7(2):282291. doi:10.1161/CIRCIMAGING. 113. 001047

17. Van Velzen JE, De Graaf FR, De Graaf MA, et al.2011 Comprehensive assessment of spotty calcifications on computed tomography angiography: Comparison to plaque characteristics on intravascular ultrasound with radiofrequency backscatter analysis. J Nucl Cardiol.18(5):893-903. doi:10.1007/s12350-011-9428-2

18. Taruya A, Tanaka A, Nishiguchi $T$, et al. 2020Lesion characteristics and prognosis of acute coronary syndrome without angiographically significant coronary artery stenosis. Eur Heart J Cardiovasc Imaging. 21(2):202-209. doi:10.1093/ehjci/jez079 


\section{Marwa Maher, et al.}

\section{تصنيف أنواع ترسبات الثرايين التاجية بالأشعة المقطعية متعددة المقاطع}

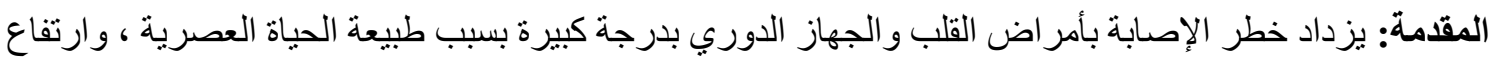

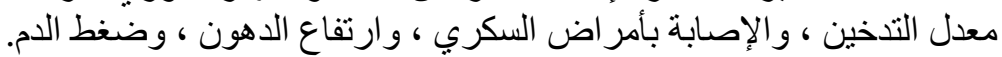

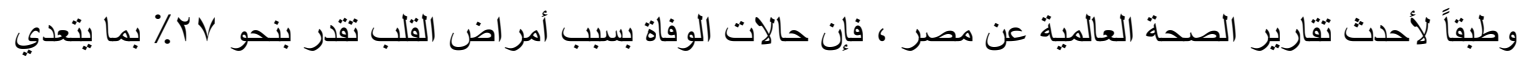

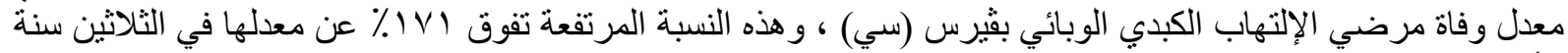
الأخيرة .

الهـف: الهدف من الرسالة ايضاح دور الاشعة المقطعية ثلاثية الابعاد فى تشخيص جلطات الثر اين التاجيةتوتحديد اذا

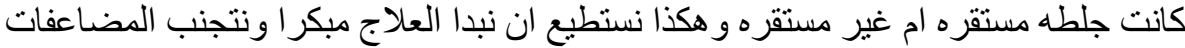
المرضى وطرق العلاج: لقد قمنا بدر اسة 7؟ مريض يعانون من جلطات في الثريان التاجية .

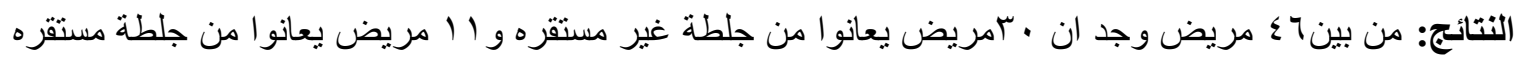

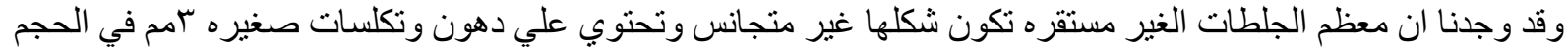

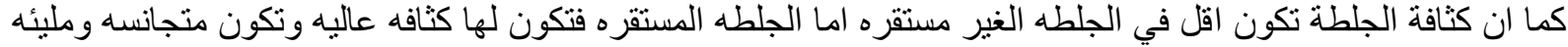

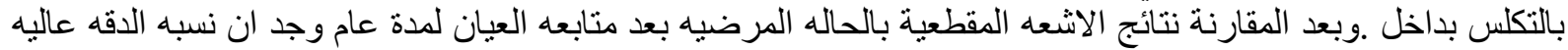
جدا فيما يتعلق بحجم التكلس وتجانس الجلطه بعد الصبغه واذا كانت الجلطه تحتوي علي الني دهون الخلاصة: الاشعة المقطعية لها دور مهم فى توصيف جلطة الثر ايين التاجية . 